\title{
Fuzzy type 1 PID controllers design for TCP/AQM wireless networks
}

\author{
Manal Kadhim Oudah, Mohammed Qasim Sulttan, Salam Waley Shneen \\ Department of Electromechanical Engineering, University of Technology, Baghdad, Iraq
}

\begin{abstract}
Article Info
ABSTRACT

Article history:

Received May 5, 2020

Revised Jul 8, 2020

Accepted Aug 2, 2020

\section{Keywords:}

Fuzzy logic control

PID controller

TCP/AQM wireless networks

The search of FLC_PID controller for TCP/AQM Wireless Networks, to deal with congestion for Internet users and to get good performance and capabilities of TCP / IP networks. Neglect of controlling the network delay and the number of continuous users that leads to a problem in the transmission process. Recently, automatic control units are adapted to solve this problem with the difficulty of controlling congestion in the presence of wireless links. This modest research presents one of the traditional PID controller methods with fuzzy logic so that wireless networks and congestion can be controlled by various configurations. The proposed methods were simulated with the required comparisons in the adoption of nonlinear systems to determine the best performance and it was found that the use of the Fuzzy logic control can achieve the best performance (reducing the delay time of delivering packets and packets loss). The simulation of the current work shows through its results the possibility of controlling the behavior of the system and through testing the balance when changed with time delay through the impact of communication time and its relationship with the stability of the work of the system.
\end{abstract}

This is an open access article under the CC BY-SA license.

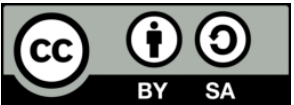

\section{Corresponding Author:}

Salam Waley Shneen

Department of Electromechanical Engineering

University of Technology

Baghdad-571-15-11, Iraq

Email: 50054@uotechnology.edu.iq

\section{INTRODUCTION}

The Quality of Service (QoS) [1], this term had become the goal that all telecom companies strive to achieve, a fulfilling this goal requires the transmission of a huge data through internet networks and that led to appear the problem of congestion of data in internet networks [2]. The congestion of network means that the number of incoming data to the router is higher than its passing data capacity that caused a big loss in transmitted packets and big delay time. Therefore, the need for the emergence of congestion control algorithms to manage internet networks become necessary to reduce the impact of this problem [3]. In the eighties of the twentieth century, the Transmission Control Protocol (TCP) $[4,5]$. It was depended as a technique to control congestion of the network. The purpose of TCP to did the end-to-end congestion control through providing the consignor with the window of congestion which holds the maximum number of unrecognized accepted packets to reduce congestion growth problem, but this technique was not enough to overcome this problem due to prompt development in the communication field. A new technique appeared that anticipate congestion before it happens. This technique is knowing as Active Queue Management algorithm (AQM) [6, 7]. It was working together with TCP technique to reduce the loss of packets and delay time in the network, to enhance the QoS. 
At the end of the last century and the beginning of this century. Many pieces of research appeared interested in a topic of AQM schemes and have been proposed many schemes of AQM, such as Random Early Detection (RED) [8], Adaptive RED (A-RED) [9]. Random Exponential Marking (REM) [10], and proportional-integral (PI) controller [11], all these schemes showed best network results with less loss of packets and less delay time through controlling queues at the bottleneck links in TCP networks. In [12], the authors (Chrysostomou, C., \& Pitsillides, A.) used a fuzzy logic control (FLC) as a control methodology to control congestion in two varied technologies: ATM and TCP/IP networks, the results of this work presented beneficial improvements in controlling congestion for ATM and TCP/IP networks. In [13] (Sen, K., Chakraborty, B., Gayen, A., \& Dey, C.), the proposed technique is Fuzzy rule-based set point weighting with a PID controller (PIDC), this technique showed a great efficacy in fulfilling important responses to the traditional PIDC used in closed-loop control applications. In [14], the authors (Bouazzi, I., Bhar, J., \& Atri, M.) are proposed to use a fuzzy logic algorithm to solve some problems that happen in Wireless sensor networks (WSNs). Such as the reliability of transmitting data preserving the latency of message, and prolonging the battery life of sensor nodes, the results showed a better power consumption compared to basic application of CSMA/CA. The presented work [15] (Mareev, N., Kachan, D., Karpov, K., Syzov, D., Siemens, E., \& Babich, Y.) discussed the problem of overload bottleneck buffers in IP networks. The authors proposed to use a new algorithm for controlling congestion such as PID-based congestion control, the simulation results appeared that the proposed algorithm was flexible, scalable, and able to averting extra queue delays resulting from loading the bottleneck buffers. The presented work [16] (Suresh, M., Hemamalini, R. R., \& Srinivasan, G. J.), proposed to use a fuzzy logic that depends on the set point weighting controller as a tuning method to overcome the problem of changing the values PID controller parameters with time or other effects.

Proportional-Integral-Derivative Controller PIDC as adaptive and proactive AQM algorithm have been proposed to reveal and control the elementary just as the present congestion adequately and proactively. The use of such as that algorithm to control congestion proactively, to make a match between the queue length and the desired level to achieve less rate of packet loss to each TCP flow to abstract the inclination against bursty sources. In this paper, we proposed to use a fuzzy logic controller (FLC) to improve the performance of AQM in TCP/IP networks, since fuzzy control improves appropriateness to dynamic system condition without a requirement for a delicate model. The proposed algorithm of fuzzy control in our work is static with a fixed fuzzy rule and fixed factors in membership functions. The current work discussed what the authors suggested using a new congestion control algorithm such as FLC, type 1- PID Controller based congestion control, and simulation results also showed that the proposed algorithm was flexible, scalable, and able to avoid additional queue delays caused by warehouse loading.

The rest of this paper is organized as follows: Section 2 contains the illustration of the TCP/AQM model and network topology, Section 3 contains the illustration of the PIDC and FLC, the simulation results clarified in Section 4, and Section 5 contains the conclusion of the paper.

\section{THE TCP/AQM MODEL AND NETWORK TOPOLOGY}

In this work, we depended on the [17-20] to illustrate the model of TCP/AQM networks, the dynamical behaviour of TCP/AQM network depends on fluid flow analysis by some stochastic differential equations with suppose the mechanism of the TCP/AQM timeout is ignored and the developed state-space model is given by the (1) and (2) [17]:

$$
\begin{aligned}
& w(t)=\frac{1}{\frac{G(t)}{C}+T_{D}}-\frac{w(t)}{2} \frac{w(t-R(t))}{\frac{G(t-R(t))}{c}+T_{D}} p(t-R(t)) \\
& \dot{q}(t)= \begin{cases}-C+\frac{w(t)}{\frac{G(t)}{C}+T_{D}} w(t) & \text { if } q(t)>0 \\
\max \left\{0,-C+\frac{N(t)}{\frac{G(t)}{C}+T_{D}} w(t)\right\} & \text { if } q(t)=0\end{cases}
\end{aligned}
$$

where:

$w^{w}(t)$ and $\dot{q}^{\dot{q}(t)}$ is the time-derivative of ${ }^{w}$ and $q$ respectively, ${ }^{w}$ is the rate of TCP window size (measured in packets). ${ }^{R}$ is the transmission of full-trip time (measured in seconds) and equal to ${ }^{\frac{q}{C}}+T_{p} ; \mathrm{q}$ is
the rate of queue length (measured in packets); ${ }^{C}$ is the capacity of the link (measured in packets/seconds); ${ }^{N}$ is the No, TCP links (load factor); ${ }^{T_{p}}$ is the promulgation delay (measured in seconds): ${ }^{p}$ is the packet sign 
probability, all these factors are assumed to be non-negative and it is the control input to reduce the transmitting rate and preserve the bottleneck queue extension.

Figure 1 shows the Flow Control Scheme and congestion avoidance in TCP/AQM networks by using (1) and (2) [20].

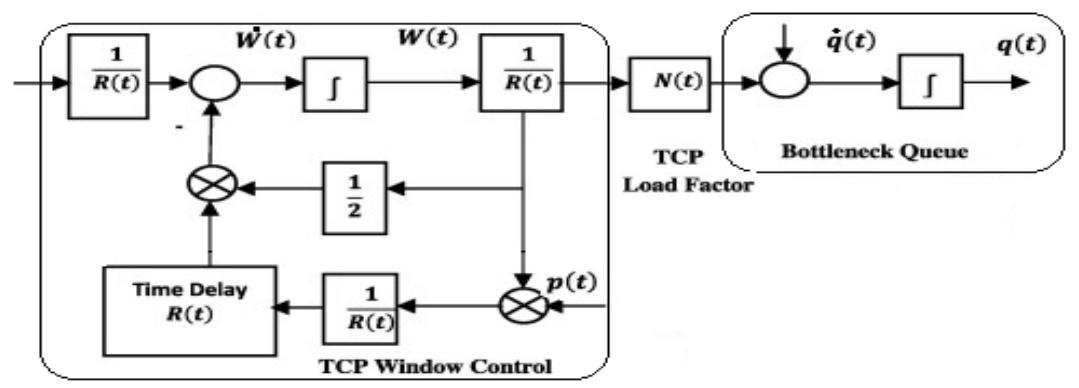

Figure 1. The flow control scheme for TCP/AQM networks

Figure 2 Shows the TCP/AQM network topology in our work, which consists of N=50. A (TCP) flow in the sender side and the same in the receiver side, two routers connected by a bottleneck are connecting by the two sides (sender and receiver). Each TCP flow and bottleneck link have link capacity equal to $15 \mathrm{Mbps}$ (3750 packets/ sec, each packet has 500 bytes). The transmission of full-trip time (Tour Time) equal to $0.218 \mathrm{sec}$, the maximum rate of queue length is 800 packets, the size of the queue is 300 packets and the promulgation delay is $5 \mathrm{msec}$, our propose controller is placed in the router 1and the droptail lay in the router 2.

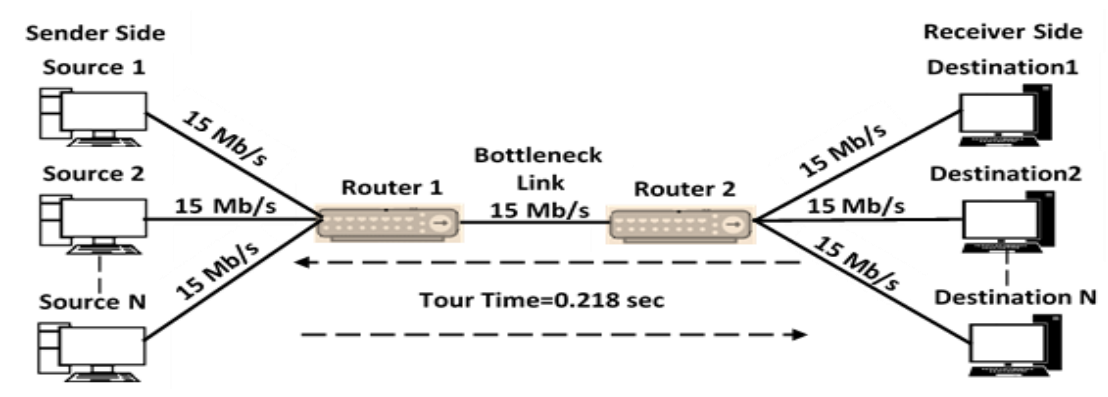

Figure 2. The network topology

\section{THE PID CONTROLLER AND FLC}

A PID controller is the most accurate and stable controller is a gadget utilized in industrial applications to control and regulate many variables of processes such as flow, pressure, and speed. To overcome the disadvantages and preserve the power of PID Controller a novel expert Fuzzy Logic Controller (FLC) has used that utilizes fuzzy logic. PID Controller utilizes a mechanism of a feedback loop to regulate variables of processes. PID Controller includes integral (I) control to improve the power of expectative for the $(\mathrm{P}+\mathrm{D})$ control. The PID Controller can remove user-level overflow, and it contains two distinctive disadvantages. Although it is widely used as an effective control to prevent overflow of the buffer. It has drawbacks that include : It is not expected, according to the design of the parameters of the controller, how to bypass the speed of the buffer speed, which wastes memory resources (wasting a large amount), which is very expensive as its use of the Internet environment becomes impractical [21].

The most conventional PID controller is described in (3):

$$
s(t)=K_{p} e(t)+K_{I} \int_{0}^{t} e(t) d t+K_{D} \frac{d e(t)}{d t}
$$

Where: 
$K_{P}, K_{I}$, and $K_{D}$ is referred to the proportional, integral, and derivative constant gain respectively. $s(t)$ Is the output of PID controller, and ${ }^{e(t)}$ is error signal between the input reference and the process output, Figure 3, shows the internal structure of PIDC with input parameters $\left(K_{P}, K_{I}, K_{D},{ }^{e}\right.$, and ${ }^{\ominus C}$ ), and with $s$ as an output parameter [22, 23].

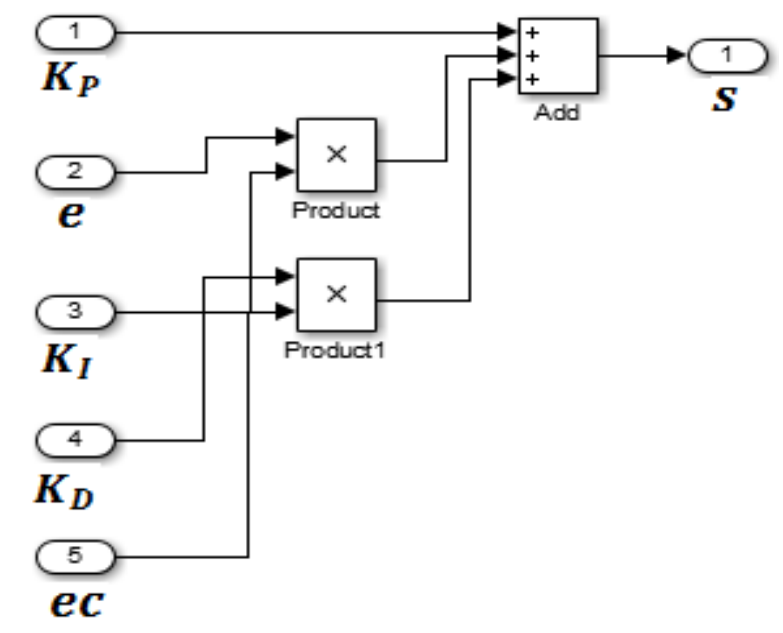

Figure 3. Simulink figure of PIDC

The control on congestion in a TCP network is considered a very complex and nonlinear process, so the fuzzy logic is used to reduce the effect of these problems, Figure 4, is shown all block diagrams that describes the fuzzy controller operation. In (4), nonlinear saturated input and time-delayed scheme:

$$
\operatorname{sat}(\mathrm{p}(\mathrm{t}-\mathrm{R}(\mathrm{t})))=\left\{\begin{array}{cc}
1, & \mathrm{p}(\mathrm{t}-\mathrm{R}(\mathrm{t})) \geq 1 \\
\mathrm{p}(\mathrm{t}-\mathrm{R}(\mathrm{t})), & 0 \leq \mathrm{p}(\mathrm{t}-\mathrm{R}(\mathrm{t}))<1 \\
0, & \mathrm{p}(\mathrm{t}-\mathrm{R}(\mathrm{t}))<0
\end{array}\right.
$$

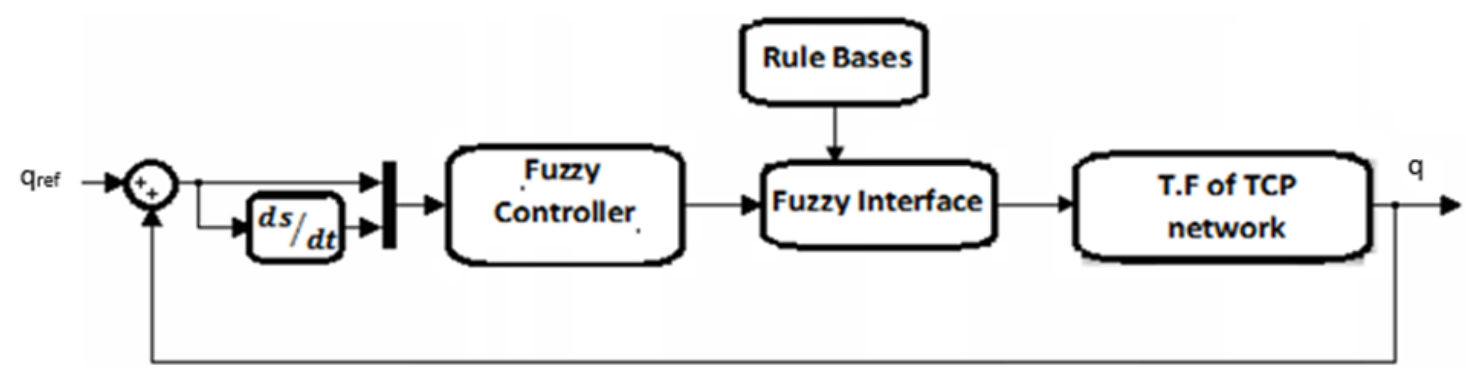

Figure 4. The block diagram of fuzzy controller operation

In this work, we arranged the fuzzy logic to subsets for all inputs and outputs (NB, NM, NS, Z, PS, PM, PB \}, where element NB refers to negative big, NM negative medium, NS negative small, Z zero, PS positive small, $\mathrm{PM}$ positive medium, and $\mathrm{PB}$ positive big. The range of functions ${ }^{e},{ }^{\ominus C}$ is $\{-6,6\}$, the range of parameter $K_{P}$ is $\{-3,4\}$, and $K_{D}, K_{I}$ is $\{-1,1\}$, Figures 5-13, Shown these ranges in Figure 5 . The simulation of FIS editor for an FLC type Mendiny, Figure 6. The surface view of the parameter $K_{D}$ in FLC, Figure 7. The simulation of rule viewer for an FLC, Figure 8. The simulation of rule editor for an FLC, Figure 9. The membership of error function ${ }^{\mathrm{I}} e^{\mathrm{m}}$ in FLC, Figure 10. The membership of change error function ${ }^{\mathrm{I}} \theta c^{\mathrm{I}}{ }$ in FLC, 
Figure 11. The membership of parameter $K_{I}$ in FLC, Figure 12. The membership of parameter $K_{P}$ in FLC and Figure 13. The membership of parameter $K_{D}$ in FLC [24-26].

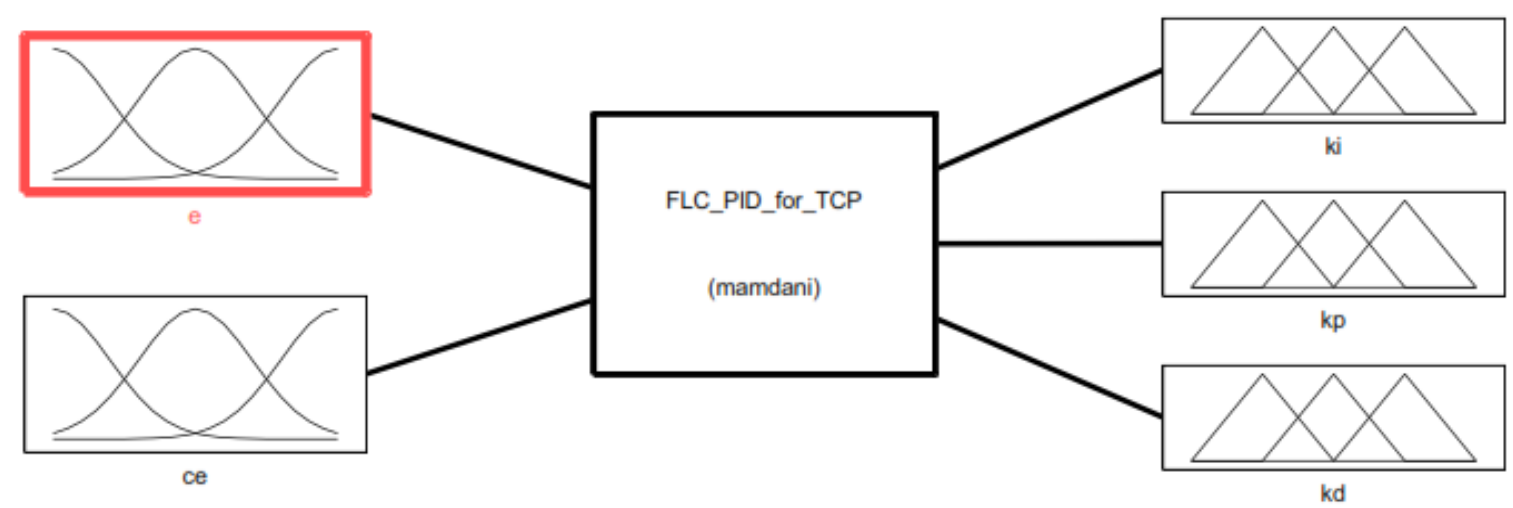

Figure 5. The simulation of FIS editor for an FLC
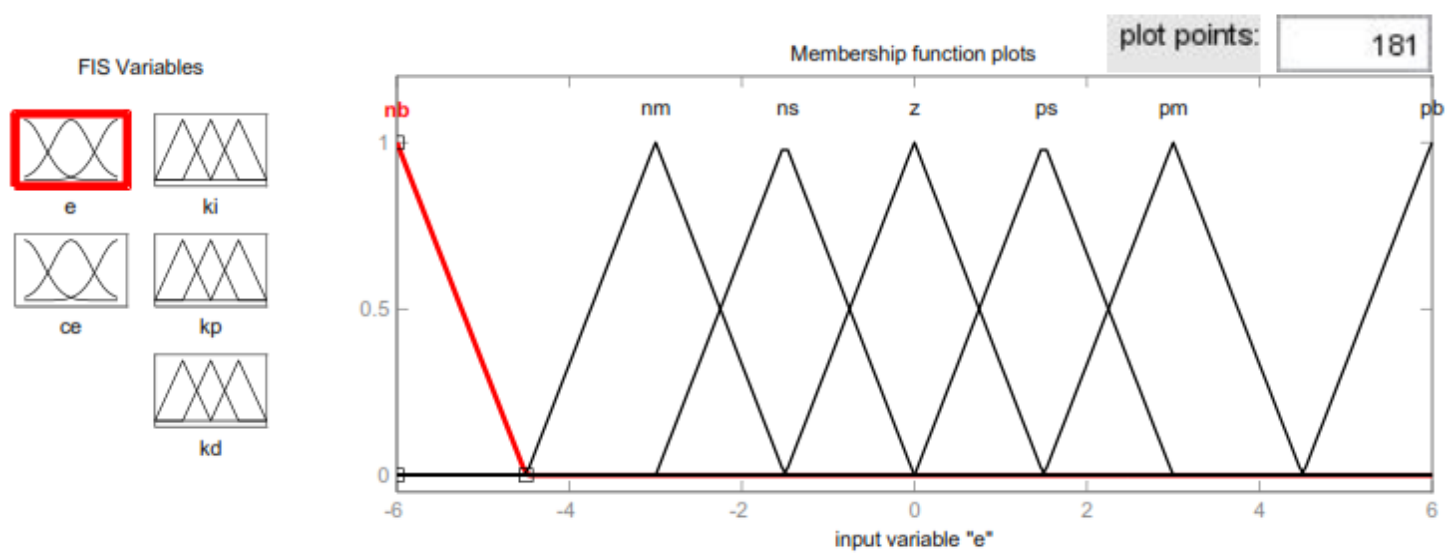

Figure 6. The membership of error function ${ }^{n} e^{n}$ in FLC
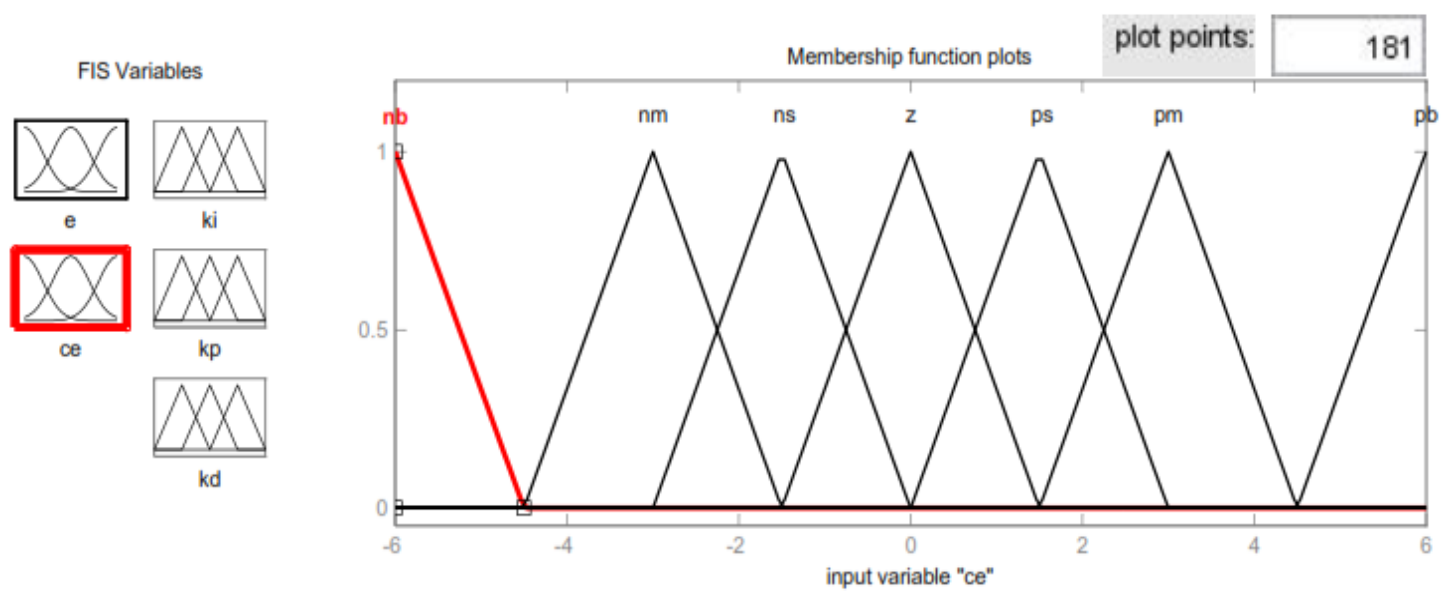

Figure 7. The membership of error function"e" in FLC 

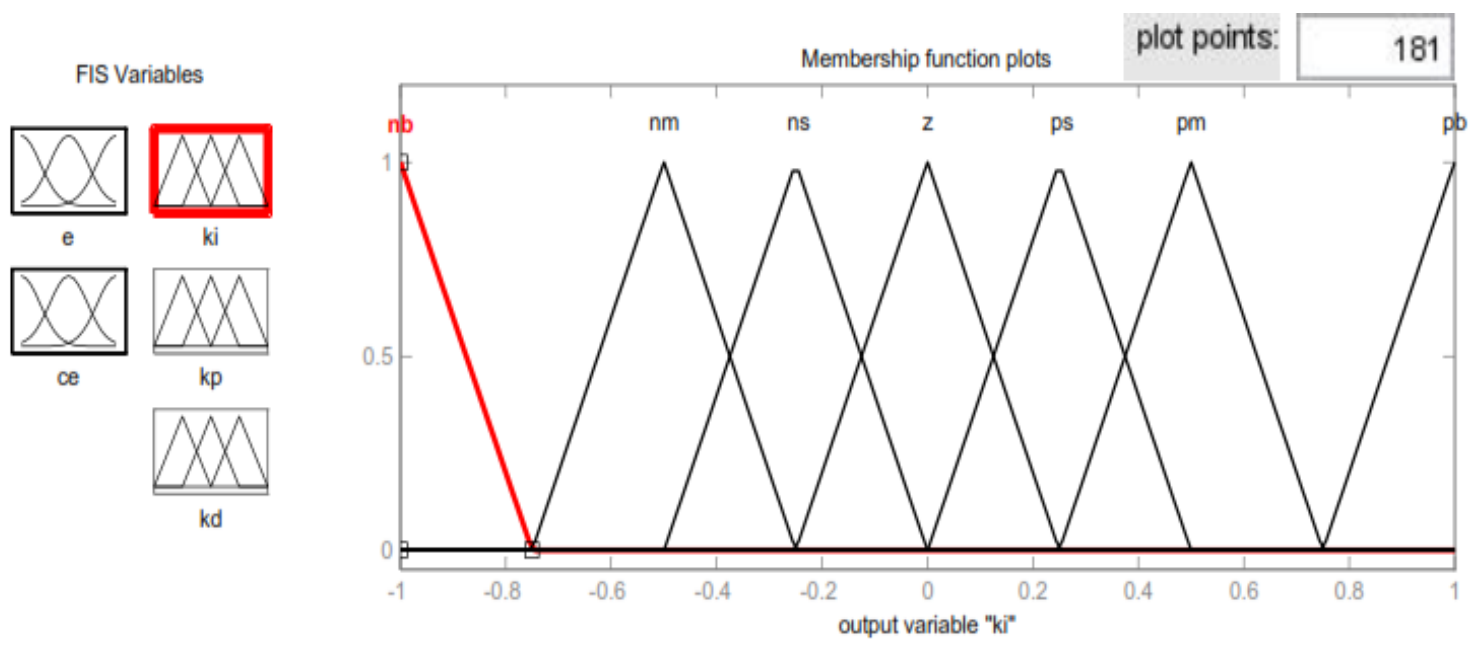

Figure 8. The membership of parameter Ki
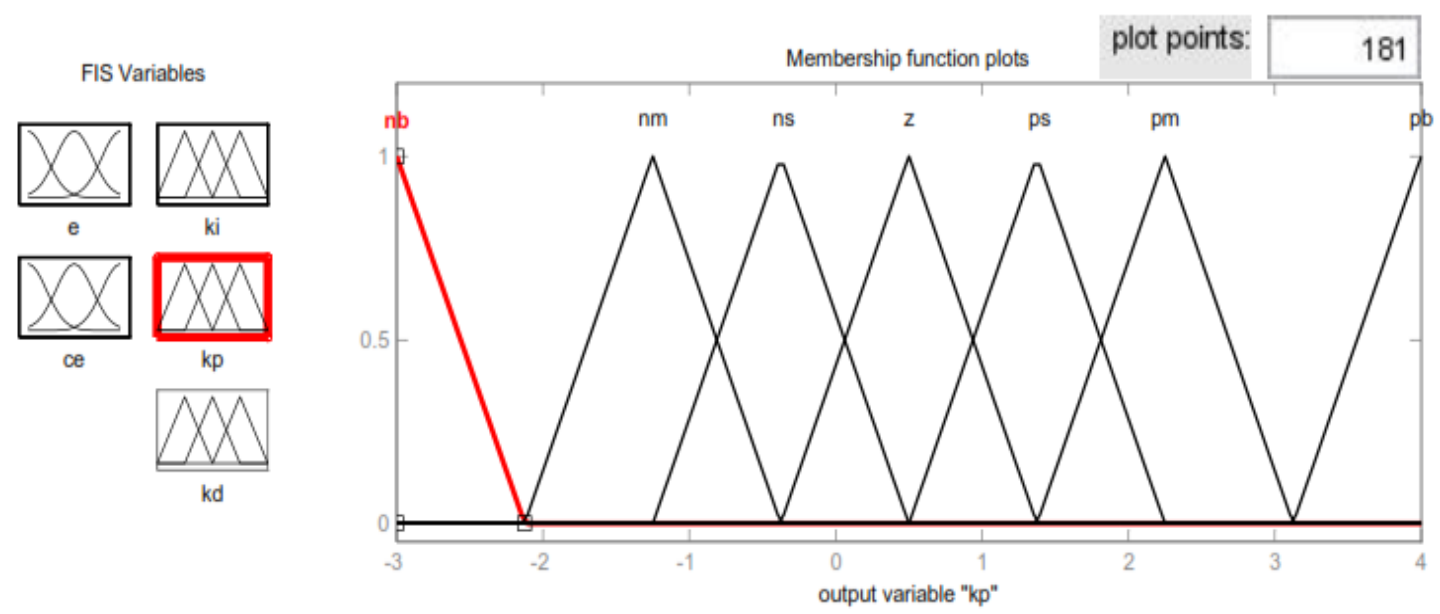

Figure 9. The membership of parameter Kp
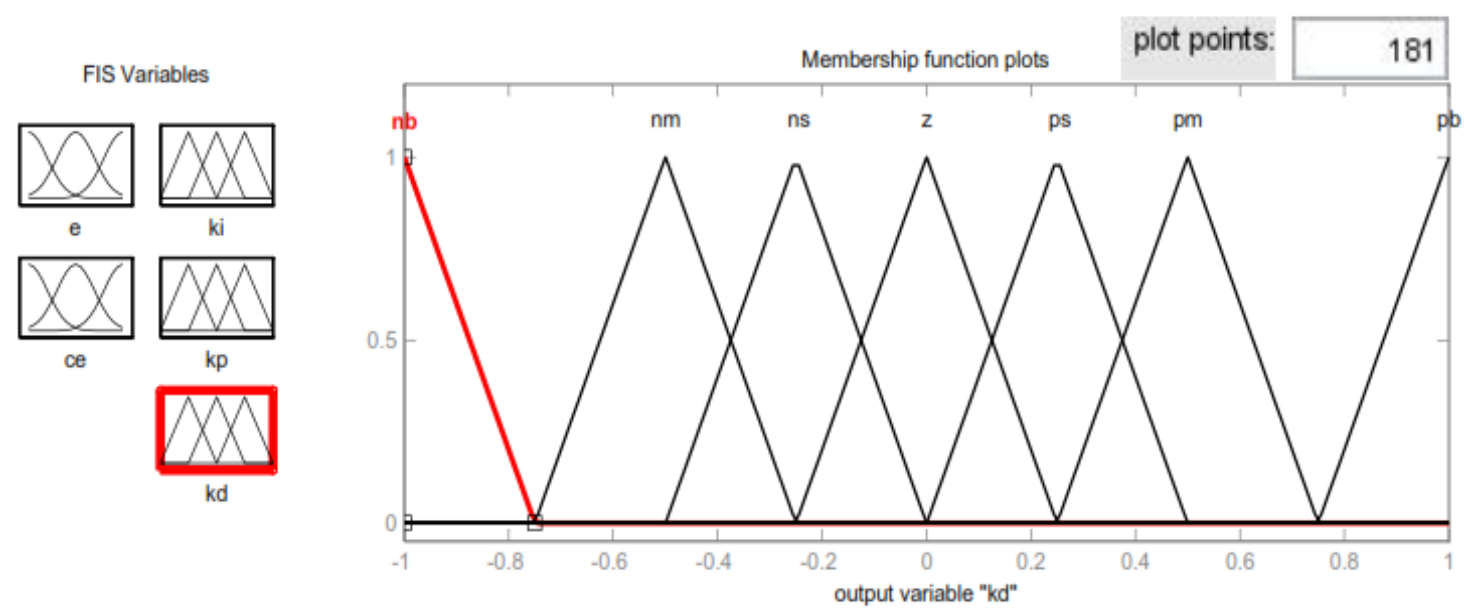

Figure 10. The membership of parameter $\mathrm{Kd}$ 


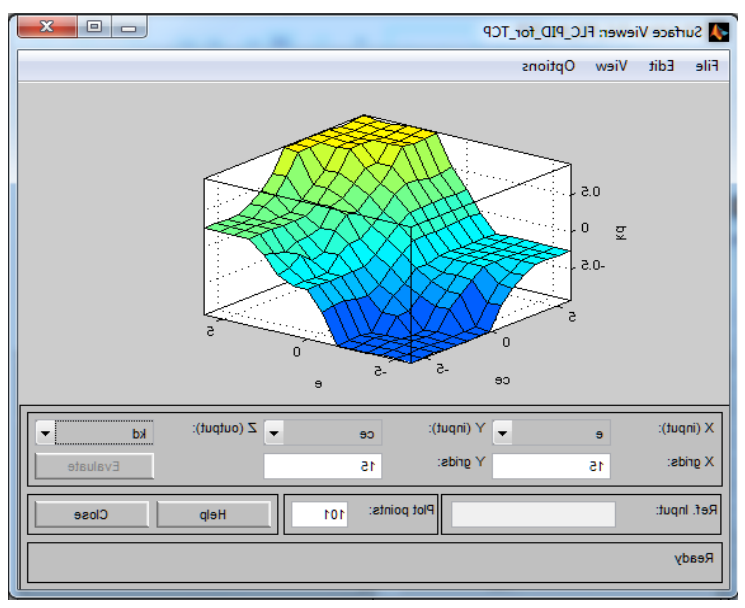

Figure 11. The surface view of the parameter $\mathrm{K}$ in FLC

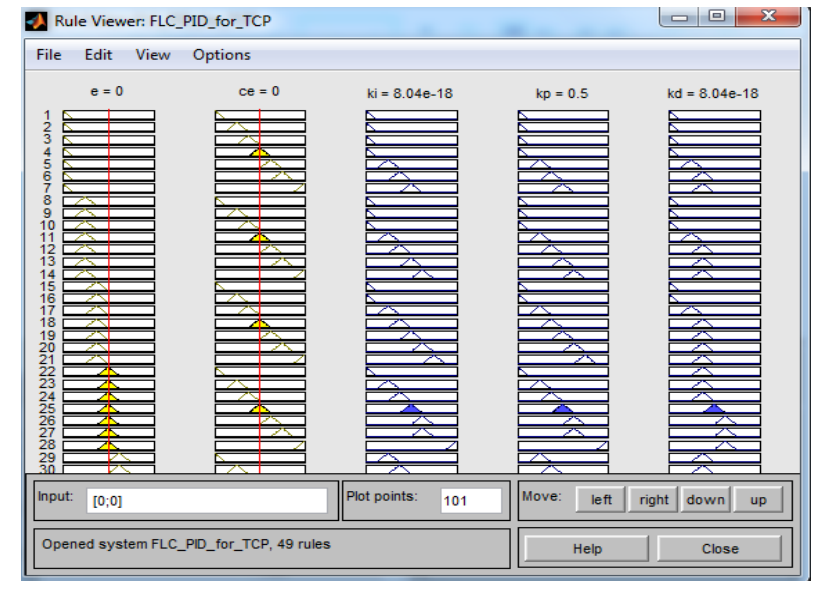

Figure 12. The simulation of rule viewer for an FLC

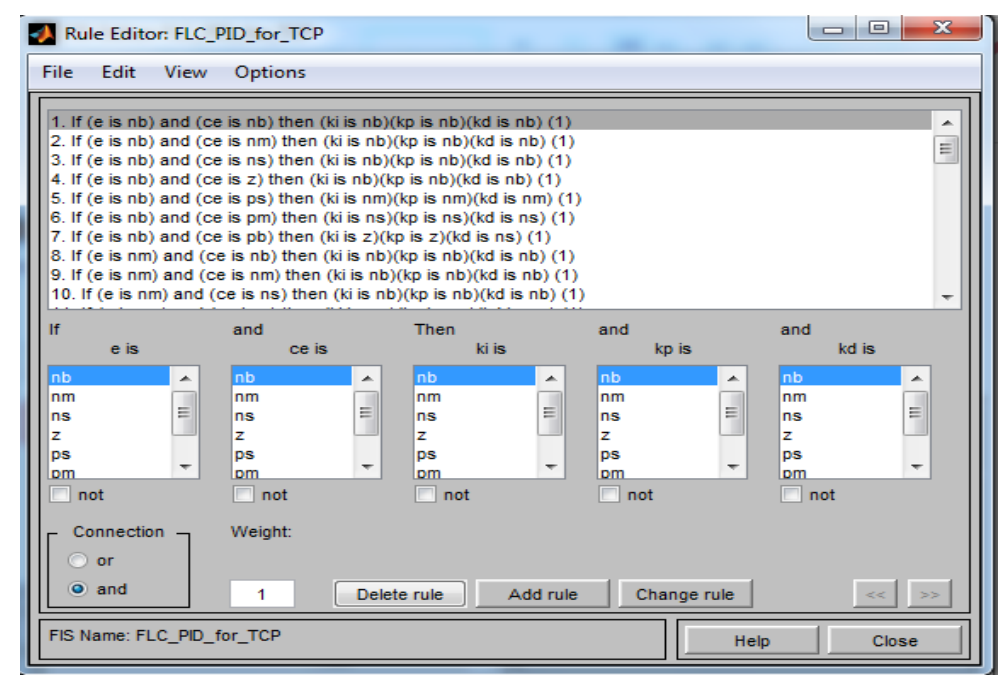

Figure 13. The simulation of rule editor for an FLC

\section{SIMULATION RESULTS}

This part, present the effectiveness of FLC with the TCP network. In addition, compression with PIDC technology to determine the best efficiency of the TCP system has made. The topic of the current study deals with improving the system performance by adopting a comparison between the two proposed types of control systems through the adoption of simulation (MATLAB) to achieve the objectives of the study to reach the efficiency of controllers and work to improve it by examining its susceptibility and avoiding network traffic. We show and discuss the simulation results for our work, the simulation results as presented in Figure 15, Figure 17 and Figure 19 exhibit what has accomplished in this work. In Figure 14. Shown the PID controller Simulink of TCP networks. It had reference value, feedback signal, PID controller and system transfer function. The step response of the TCP network with the action of the PID controller has appeared in Figure 16. Also, in Figure 18 shown the FLC-PID Simulink of TCP networks. The step response of TCP network with the action of PIDC has appeared in Figure 15 and in Figure 17 shown the Simulink diagram of PID controller and FLC-PID of TCP networks to compare the steps response between two types of controllers. In Figure 19 illustrates the step response when compare between FLC-PID and PIDC for TCP networks.

The simulation results present the percentages of those results that achieved from proposed work. like rising time (PID controller at 0.0146, FLC- PID controller at 0.00672) and settling time (PID controller at 0.189 , FLC- PID controller at 0.124 ) with error overshoot (PID controller at 0.0728, FLC- PID controller at 0.03 , where minimizing these percentages resulting in higher utilization from the queue usage. 


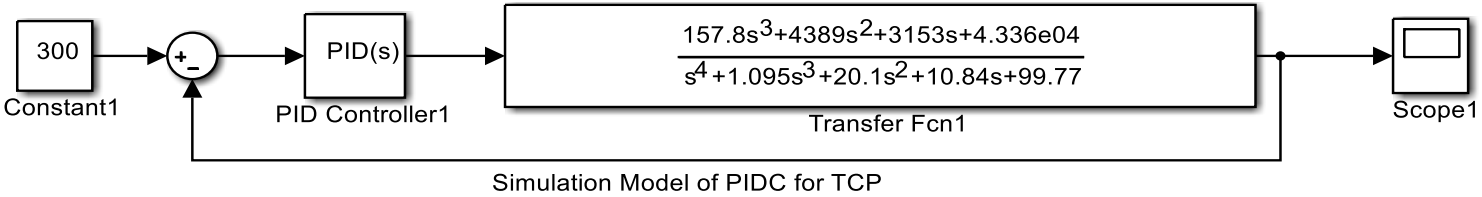

Figure 14. The PIDC Simulink form of TCP networks

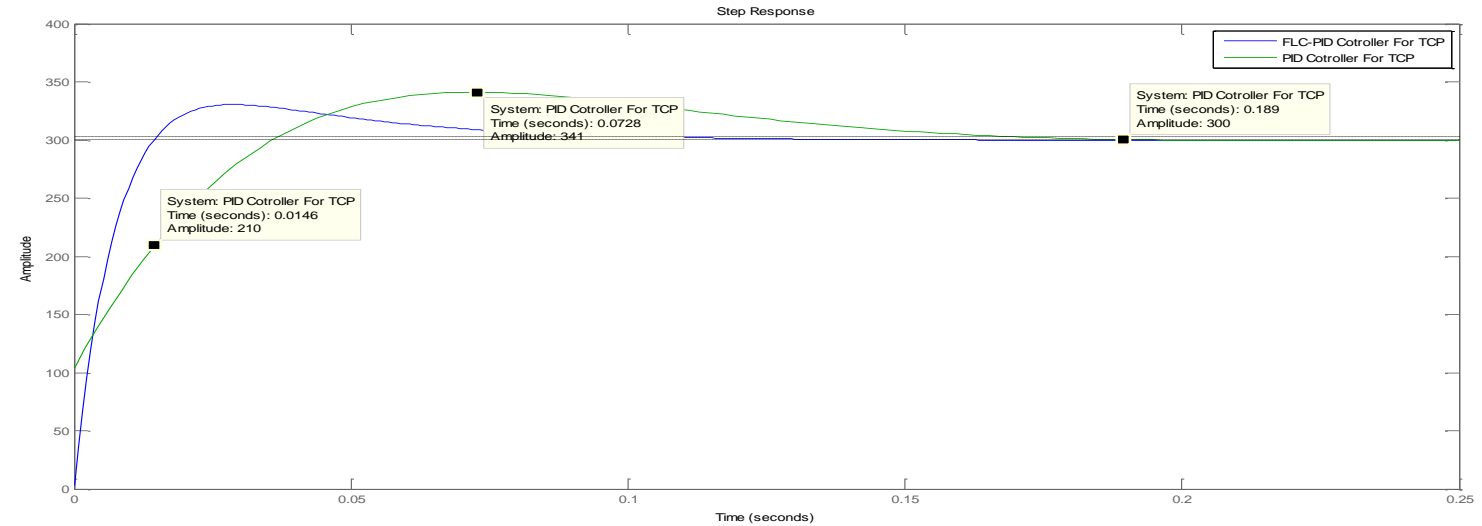

Figure 15. The Simulation results step response of TCP network with PIDC

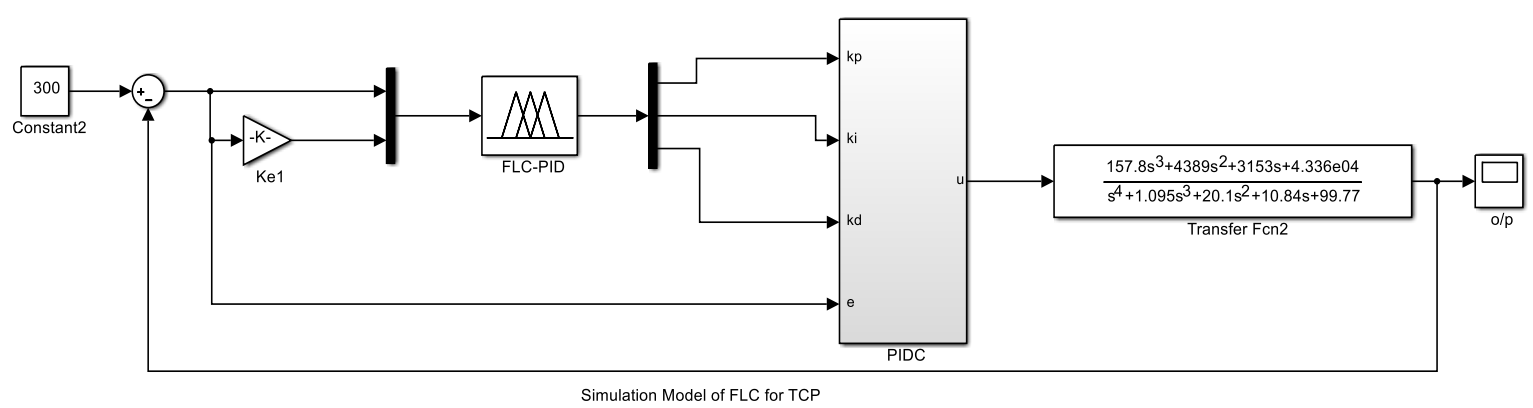

Figure 16. The FLC-PID Simulink form of TCP networks

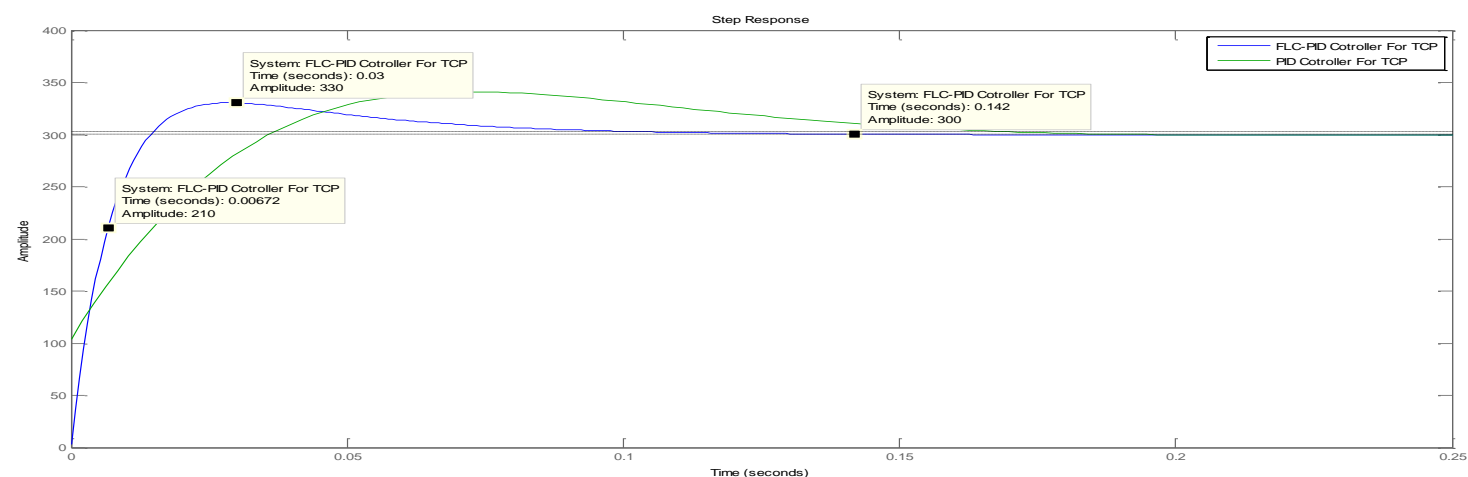

Figure 17. The step response of TCP network with FLC-PID 


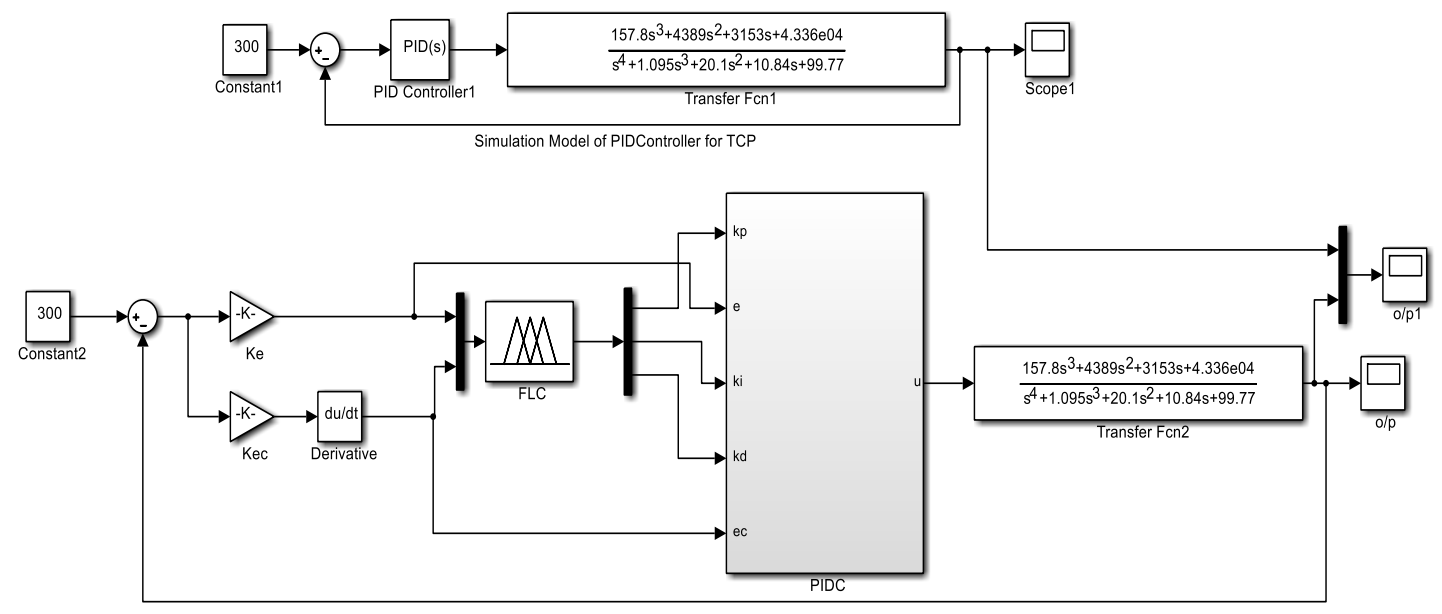

Simulation Model of FLC- PIDController for TCP

Figure 18. Simulink diagram for PIDC and FLC-PID of TCP networks

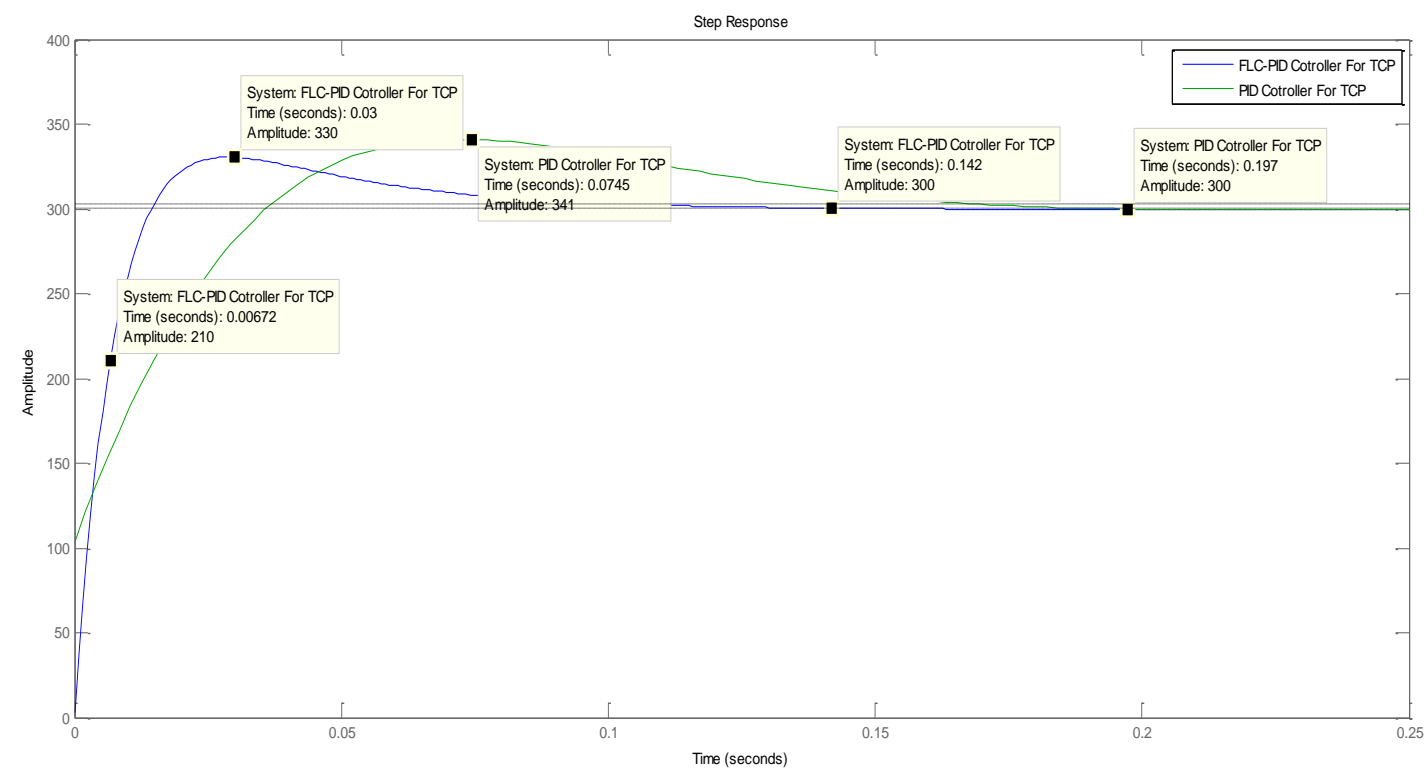

Figure 19. The step response for PIDC and FLC-PID of TCP network with

\section{CONCLUSION}

The current work is a comparison of two types of control systems and the chosen application is a TCP system. A traditional PID controller plus PID-fuzzy. Through simulation to obtain results, it shows performance and observes the difference in response. The results indicated that the PID-fuzzy was more stable. The work had designed within certain working conditions so that there would be a controller that interferes in design to obtain performance within the same conditions of the proposed system and improve performance through the speed of response to the control unit and the stability of the system. In this paper, two PID \& FLC-PID controllers are proposed with AQM for TCP network to avoid network congestion. By simulating the proposed FLC-PID, good results (as mention in Section 4) were obtained compared to the PID by such as stability time, faster rise time and a lower percentage of overtaking.

As a future work:

a) Using a newly optimization algorithm for optimizing the controller's gains based on an objective function, where the manual adaptation does not reach the required error minimization.

b) The optimization algorithm can be PSO, ABC, ant, antlion, WOA, lions, bats, fireflies 


\section{REFERENCES}

[1] Khudhair, A. A., Jabbar, S. Q., Sulttan, M. Q., \& Wang, D. "Wireless indoor localization systems and techniques: survey and comparative study," Indonesian Journal of Electrical Engineering and Computer Science, vol. 3, no. 2, pp. 392-409, 2016.

[2] Sulttan, M. Q. "Reducing Computational Complexity and Enhancing Performance of IKSD Algorithm for Uncoded MIMO Systems," Indonesian Journal of Electrical Engineering and Computer Science, vol. 2, no. 3, pp. 636-646, 2016.

[3] He, K., Rozner, E., Agarwal, K., Gu, Y., Felter, W., Carter, J., \& Akella, A. “AC/DC TCP: Virtual congestion control enforcement for data centre networks," In Proceedings of the 2016 ACM SIGCOMM Conference, pp. 244-257, Aug 2016.

[4] Ren, Y., Li, J., Shi, S., Li, L., Wang, G., \& Zhang, B. "Congestion control in named data networking-a survey," Computer Communications, vol. 86, pp. 1-11, 2016.

[5] Leong, W. K., Wang, Z., \& Leong, B. "TCP congestion control beyond bandwidth-delay product for mobilecellular networks," In Proceedings of the 13th International Conference on emerging Networking EXperiments and Technologies, pp. 167-179, Nov 2017.

[6] Hamidian, H., \& Beheshti, M. T. "A robust fractional-order PID controller design based on active queue management for TCP network,” International Journal of Systems Science, vol. 49, no. 1, pp. 211-216, 2018.

[7] Liu, Y., Liu, X., Jing, Y., Zhang, Z., \& Chen, X. "Congestion tracking control for uncertain TCP/AQM network based on integral backstepping," ISA transactions, vol. 89, pp. 131-138, 2019.

[8] Floyd, S., \& Jacobson, V. "Random early detection gateways for congestion avoidance," IEEE/ACM Transactions on Networking, vol. 1, no. 4, pp. 397-413, 1993.

[9] Floyd, S., Gummadi, R., \& Shenker, S. “Adaptive RED: An algorithm for increasing the robustness of RED's active queue managemen," under submission, 2001.

[10] Athuraliya, S., Low, S. H., Li, V. H., \& Yin, Q. "REM: Active queue management," IEEE Network, vol. 15, no. 3, pp. 48-53, 2001.

[11] Hollot, C. V., Misra, V., Towsley, D., \& Gong, W. "Analysis and design of controllers for AQM routers supporting TCP flows," IEEE Transactions on automatic control, vol. 47, no. 6, pp. 945-959, 2002.

[12] Chrysostomou, C., \& Pitsillides, A. "Congestion control in computer networks using fuzzy logic," In Proceedings of the 10th WSEAS International Conference on Communications, ICCOM, vol. 6, pp. 551-556, Jul 2006.

[13] Sen, K., Chakraborty, B., Gayen, A., \& Dey, C. "Fuzzy Rule-Based Set Point Weighting for PID Controller," In Advances in Communication, Devices and Networking, pp. 797-806, Springer, Singapore, 2018.

[14] Bouazzi, I., Bhar, J., \& Atri, M. "Priority-based queuing and transmission rate management using a fuzzy logic controller in WSNs," ICT Express, vol. 3, no. 2, pp. 101-105, 2017.

[15] Mareev, N., Kachan, D., Karpov, K., Syzov, D., Siemens, E., \& Babich, Y. "Efficiency of a PID-based Congestion Control for High-speed IP-networks," In Titel: Proceedings of the 6th International Conference on Applied Innovations in IT. Bibliothek, Hochschule Anhalt, Sep 2018.

[16] Suresh, M., Hemamalini, R. R., \& Srinivasan, G. J. "Fuzzy Logic Based Set-Point Weighting Controller Tuning for an Internal Model Control Based PID Controller," Sensors \& Transducers, vol. 109, no. 10, pp. 29, 2009.

[17] Hollot, C. V., Misra, V., Towsley, D., \& Gong, W. B. "A control theoretic analysis of RED, In Proceedings IEEE INFOCOM 2001. Conference on Computer Communications. Twentieth Annual Joint Conference of the IEEE Computer and Communications Society (Cat. No. 01CH37213), vol. 3, pp. 1510-1519, IEEE, Apr 2001.

[18] Marami, B., \& Haeri, M. "Implementation of MPC as an AQM controller," Computer Communications, vol. 33, no. 2, pp. 227-239, 2010.

[19] Azuma, T., Fujita, T., \& Fujita, M. "A design of state predictive Hœ congestion controllers for TCP/AQM networks," Transactions of the Institute of Systems, Control and Information Engineers, vol. 18, no. 10, pp. 373-375, 2005.

[20] Bisoy, S. K., \& Pattnaik, P. K. "Design of feedback controller for TCP/AQM networks," Engineering Science and Technology, an International Journal, vol. 20, no.1, pp. 116-132, 2017.

[21] Wong, A. K., Lin, W. W., Ip, M. T., \& Dillon, T. S. "Genetic algorithm and PID Control together for dynamic anticipative marginal buffer management: an effective approach to enhance dependability and performance for distributed mobile object-based real-time computing over the internet," Journal of Parallel and Distributed Computing, vol. 62, no. 9, pp. 1433-1453, 2002.

[22] Attiya, A. J., Shneen, S. W., Abbas, B. A., \& Wenyu, Y. "Variable Speed Control Using Fuzzy-PID Controller for Two-phase Hybrid Stepping Motor in Robotic Grinding," Indonesian Journal of Electrical Engineering and Computer Science, vol. 3, no. 1, pp. 102-118, 2016.

[23] Shneen, S. W., Sulttan, M. Q., \& Jaber, M. H. "Variable speed control for 2Ph-HSM in RGS: a comparative simulation study," International Journal of Electrical and Computer Engineering, 10, no. 3, pp. 2285, 2020.

[24] Madrigal, Gilfred Allen M., et al., "Fuzzy logic-based maximum power point tracking solar battery charge controller with backup stand-by AC generator," Indonesian Journal of Electrical Engineering and Computer Science," vol. 16, no. 1, 2019.

[25] Farhan, Mohd Faisal, et al., "A simplify fuzzy logic controller design based safe experimentation dynamics for Pantograph-Cateary system," Indonesian Journal of Electrical Engineering and Computer Science, vol. 14, no. 2, pp. 903-911, 2019.

[26] Handayani, Ade Silvia, et al., "Analysis on swarm robot coordination using fuzzy logic," Indonesian Journal of Electrical Engineering and Computer Science (IJEECS), vol. 13, no. 1, pp. 48-57, 2019. 